\title{
Nuclear Receptor 5A2 Regulation of Agrp underlies Olanzapine-induced Hyperphagia
}

\section{Running title: Nr5a2 drives antipsychotic-induced food intake}

Rizaldy C. Zapata ${ }^{a}$, Dinghong Zhanga ${ }^{a}$ Avraham Libster ${ }^{\mathrm{a}}$, Alessandra Porcu ${ }^{\mathrm{b}}$, Patricia Montilla-Perez ${ }^{\mathrm{a}}$, Zhi Zhang $^{\mathrm{c}}$, Stephanie M Correa ${ }^{\mathrm{c}}$, Francesca Telese ${ }^{\mathrm{a}}$, Olivia Osborn ${ }^{\mathrm{a} *}$

aDivision of Endocrinology and Metabolism, Department of Medicine, University of California San Diego, La Jolla, CA, 92093

${ }^{b}$ Department of Psychiatry and Center for Circadian Biology, University of California San Diego, La Jolla, CA, 92093

'Department of Integrative Biology and Physiology, University of California Los Angeles, Los Angeles, CA, 90095

${ }^{*}$ Corresponding Author:

Dr. Olivia Osborn

Division of Endocrinology and Metabolism

University of California San Diego

9500 Gilman Drive Mail Code 0673

La Jolla, California 92093 USA

oosborn@ucsd.edu 


\section{Summary/Abstract}

Antipsychotic (AP) drugs are highly efficacious treatments for psychiatric disorders, but a serious side effect of their use is excessive weight gain and subsequent development of metabolic disease. Increased food intake is the underlying driver of AP-induced weight gain, although the underlying mechanisms remain unknown. In previous studies, we identified hypothalamic genes whose expression level was altered following APs-induced hyperphagia. Among these genes, the orexigenic peptide Agrp and the transcription factor nuclear receptor subfamily 5 group A member 2 (Nr5a2) were two of the most significantly upregulated genes by APs. NR5a2 is broadly expressed throughout the body, but little is known about its role in the brain. In this study, we investigated the role of hypothalamic NR5a2 in AP-induced hyperphagia and weight gain. In hypothalamic cell lines, OLZ treatment resulted in a dose-dependent increase in gene expression of NR5a2 and Agrp. In mice, administration of a specific $\mathrm{Nr} 5 \mathrm{a} 2$ inhibitor decreased olanzapine-induced hyperphagia and weight gain, while knockdown of $\mathrm{Nr} 5 \mathrm{a} 2$ in the arcuate nucleus (ARC) partially reversed olanzapine-induced hyperphagia. Chromatinimmunoprecipitation-PCR studies showed for the first time that NR5a2 directly binds to the Agrp promoter region. In addition, in situ hybridization studies confirm that NR5a2 and Agrp are co-localized in a subset of cells in the arcuate nucleus. In summary, we identify $\mathrm{Nr} 5 \mathrm{a} 2$ as a key mechanistic driver of AP-induced food intake and these findings can be used to inform future clinical development of APs that do not activate hyperphagia and weight gain. 


\section{INTRODUCTION}

Antipsychotic (AP) medications are highly efficacious treatments for various psychiatric disorders ${ }^{1-6}$ but a serious side effect of their use is excessive weight gain ${ }^{3,7,8}$. Approximately $20 \%$ of patients treated with a broad range of APs gain clinically significant amounts of weight $(>7 \% \text { of their baseline weight })^{9}$. Drug safety reviews have shown the percentage of patients gaining a clinically significant amount of weight varies between individuals and depending on the drug, ranging from $\sim 20-40 \%$ for olanzapine (OLZ) and clozapine and $\sim 10-20 \%$ for quetiapine and risperidone ${ }^{9-16}$. While OLZ is associated with a very high risk for weight gain, it is also regarded as one of the most clinically effective medications ${ }^{17}$. APs induce weight gain in both human ${ }^{7,18-20}$ and rodents ${ }^{21-26}{ }^{27-30}$, by increasing food intake (hyperphagia). Despite widespread efforts to understand how APs induce hyperphagia, very little is known about the mechanisms underlying this serious adverse effect. Previous studies have relied on non-specific anti-obesity drugs that suppress basal feeding to reduce AP-induced weight gain (i.e. locaserin ${ }^{31}$, orlistat $^{32}$, liraglutide ${ }^{33}$, nizatidine ${ }^{34}$ metformin $^{35}$ ). While using anti-obesity drugs in combination with APs is clinically beneficial to offset weight gain, they do not shed light on the specific mechanisms underlying APinduced hyperphagia. Delineating the specific mechanisms driving AP-induced hyperphagia can be used to inform future drug development of highly effective APs without this metabolic liability and also more broadly to understand pathways regulating food intake that could be used as potential anti-obesity strategies.

In our previous work, we used a $C$. elegans-based high-throughput screen ${ }^{36}$ to identify specific chemical suppressors of AP-induced hyperphagia ${ }^{27}$. We then conducted studies in a well-established mouse model of AP-induced hyperphagia and weight gain ${ }^{26-31,37-40}$ to determine whether the compounds identified in the $C$. elegans screen could also suppress AP-induced hyperphagia in mammals ${ }^{27}$. These studies identified hypothalamic genes whose transcriptional levels were associated with APs-induced hyperphagia, which include the orexigenic peptide Agrp and the transcription factor nuclear receptor subfamily 5 group A member 2 (Nr5a2), among the most significantly upregulated genes by APs. Food intake is regulated by many parts of the brain ${ }^{41}$ and agouti-related peptide expressing (Agrp) neurons in the arcuate nucleus (ARC) of the hypothalamus play a

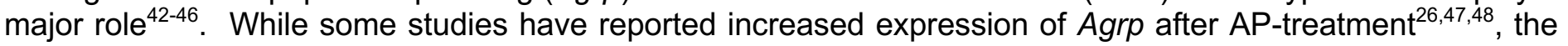
importance of this neuronal subtype and the molecular mechanisms regulating the AP-induced expression of this key pro-feeding gene are not well understood ${ }^{49-51}$. Furthermore, the hypothalamic upregulation of Nr5a2 is specifically associated with AP treatment and not increased body weight, suggestive of its important role in APinduced hyperphagia ${ }^{27}$.

Nr5a2, also called Liver receptor homolog -1 (LRH-1), is best known for its role in the periphery and governs a transcriptional network of genes involved in bile acid signaling and liver lipid homeostasis ${ }^{52-54}$. Nr5a2 has also been implicated in adipocyte formation ${ }^{55}$, intestinal function ${ }^{56}$ pancreatic inflammation ${ }^{57}$ and expression of pancreatic digestive enzymes ${ }^{58,59}$. However, $\mathrm{Nr} 5 \mathrm{a} 2$ is also widely expressed in the mouse brain ${ }^{60}$ where it controls neural stem cell fate ${ }^{61}$. Within the brain, Nr5a2 is expressed in the ARC of the hypothalamus ${ }^{62-64}$ and more recently has been shown to mark a select subset of neurons in this region ${ }^{65}$ but little is known about its role in the central nervous system $(\mathrm{CNS})^{61,64}$. Our previous studies provided the first insights into the potential involvement of NR5a2 in AP-induced food intake. In these C.elegans based studies, we determined that NR5a2 ortholog/nhr-25 mutant strain (nhr-25(ku215)) was resistant to AP-induced hyperphagia ${ }^{27}$. NR5a2 is broadly expressed throughout the body and has well-described roles in the liver ${ }^{54,66}$, gut ${ }^{56}$, and pancreas ${ }^{57,67}$ but little is known about its role in the $\mathrm{CNS}^{61,64}$. In the current study, we used several mouse models to investigate the role of NR5a2 in antipsychotic-induced food intake and weight gain.

\section{MATERIALS AND METHODS}

In vitro studies: Adult mouse hypothalamic cell lines (mHypoA-59, CLU468 cells, Cedarlane) were cultured as described previously ${ }^{68,69}$. In brief, cells were grown and maintained in high-glucose, pyruvate-free DMEM supplemented with $10 \%$ fetal bovine serum, L-glutamine (Cat. 25030081, Gibco, NY), and $10 \mathrm{u} / \mathrm{ml}$ of penicillin and $10 \mathrm{ug} / \mathrm{ml}$ of streptomycin (Cat. 15149-122, Gibco) of in a 5\% CO $\mathrm{CO}_{2}$ environment. Cells were treated with OLZ $(25 \mu \mathrm{M}-200 \mu \mathrm{M})$ for 6 hours and then RNA extracted for gene expression analysis. Cells were also co-treated 
with NR5a2 antagonist SR1848 (Aobious, Gloucester, MA) for 6 hours at $1-5 \mu M^{70}$ in DMSO as described previously.

Gene expression: RNA isolation was performed using Trizol (cat \# 15596026, Invitorgen) and was purified using RNeasy Plus Mini Kit (cat \# 774104, Qiagen) using the manufacturer's recommendations. cDNA was reverse transcribed from 300 ng of RNA using High Capacity cDNA transcription kit (cat \# 4368813, Applied Biosystems). Relative expression analyzed by qPCR using StepOne Realtime PCR System. Gene expression was calculated after normalization to the housekeeping genes ${ }^{71}$ using the $\Delta^{\Delta \mathrm{Ct}}$ method. Gene expression was calculated relative to experimental controls. Primer sequences (5'-3') used to measure gene expression are listed in Table 1.

Mice

All protocols were approved by UCSD IACUC. All mice were singly housed in standard cages and acclimated to laboratory conditions (12:12 light-dark, $20-21^{\circ} \mathrm{C}, 50 \%$ humidity) for 7 days before experimentations. Mice were singly housed to allow accurate measurement of daily food intake by weighing food in the hopper and accounting for any spillage ${ }^{72}$. All studies were performed in female C57B6/J mice (Jackson, stock \# 000664) or Agrp null mice. Agrp $^{-/-}$mice were gifted by Dr. Chen Liu of UT Southwestern.

Olanzapine administration: Olanzapine (OLZ) was compounded into 45\% HFD diet (54 mg/kg = 6-8mg/kg) as a convenient dosing strategy ${ }^{27,29,30}$, and this approach has been used in many other studies investigating APinduced hyperphagia and weight gain ${ }^{26,37-40}$. This dose results in mouse plasma levels $(21 \pm 5 \mathrm{ng} / \mathrm{ml})$ that are similar to levels observed in humans treated with OLZ $(10-50 \mathrm{ng} / \mathrm{mL})^{38}$.

Systemic inhibition of $\mathrm{Nr} 5 \mathrm{a} 2$. Twelve-week old female mice were acclimated to receive intraperitoneal (IP) injections of sterile saline for 3 days before the experiment and then were randomized to receive a $45 \%$ high fat diet (CON, D09092903B, Research Diets) with or without olanzapine (OLZ, 54mg/kg, D16111030). Mice were then further randomized to receive the vehicle solution (VEH, 10\% DMSO, 10\% Tween 80 in $0.9 \% \mathrm{NaCl}$ ) or the $\mathrm{Nr} 5 \mathrm{a} 2$ inhibitor (SR1848) at $30 \mathrm{mg} / \mathrm{kg}$ daily for 7 days. Food intake and body weight were measured daily. Animals were sacrificed at the end of the study and the hypothalamus was dissected, snap frozen in liquid nitrogen, and stored in $-80^{\circ} \mathrm{C}$ until analyses.

Hypothalamic inhibition of Nr5a2. Twelve-week old female mice were anesthetized with isoflurane and were mounted on a heating pad on a Neurostar robotic stereotaxic surgery set-up. Nr5a2 siRNA (SMARTpool: Accell, Catalog ID:L-047044-01-0005, Dharmacon, Lafayette,CO) or non-targeting control siRNA (Catalog \# K-nin; Dharmacon, Lafayette,CO) ( $\mathrm{n}=4-5)$ was delivered bilaterally into the ARC using coordinates: A-P: $-1.58 \mathrm{~mm}$ from Bregma; $M-L \pm 0.25 \mathrm{~mm}$ from midline; $\mathrm{D}-\mathrm{V}:-5.8 \mathrm{~mm}$ into the skull. Mice were allowed to recover for 7 days before transitioning to CON or OLZ. Food intake was measured daily and body weight every other day for 14 days. Animals were sacrificed at the end of the study and the hypothalamus was dissected, snap frozen in liquid nitrogen, and stored in $-80^{\circ} \mathrm{C}$ until analyses.

Agrp null studies. Twelve-week old WT and Agrp $^{-1-}$ female mice were randomized to receive either CON or OLZ ( $n=9-17 / g r o u p)$. Food intake was measured daily and body weight every other day for 12 days. Animals were sacrificed at the end of the study and the hypothalamus was dissected, snap frozen in liquid nitrogen, and stored in $-80^{\circ} \mathrm{C}$ until analyses.

Chromatin Immunoprecipitation (ChIP). ChIP experiments were conducted in triplicates using methods previously described in other neuronal cell types ${ }^{73-75}$. Briefly, hypothalamic mHypoA-59 cells were grown in $10 \mathrm{~cm}$ dishes and at $75-80 \%$ confluency and fixed with $1 \%$ formaldehyde. Nuclei were isolated before chromatin extraction. Chromatin from approximately 10 million cells was sheared using a sonication device (Bioruptor Pico, \#2013-2019, Diagenode) and optimized to produce $~ 400$ bp fragments. Chromatin was immunoprecipitated using 4ug of Nr5a2 antibody (PP-H2325-00. $5 \mu \mathrm{g} /$ ChIP, RD Biosystems) ${ }^{57}$ and 20ul of beads without any antibody were used as control sample. Importantly, this antibody has been validated in the $\mathrm{Nr} 5 \mathrm{a} 2 \mathrm{KO}^{52}$ and has successfully been used in liver ${ }^{76,77}$ and pancreatic ${ }^{57}$ ChIP experiments in mice. After primary and secondary antibody incubation and washes, purified DNA was used in quantitative PCR reactions with primers targeting the promoter of NR5a2 target gene Prospero Homeobox 1(Prox1) promoter (Prox1-F 5'CTGTTAACTGTGCCCAGgGAGAGGA-3', Prox1-R 5'-TGGTTTGACATCTTGGGTGA-3') ${ }^{61}$ as a positive 
control $^{61}$ or the Agrp promoter region (Agrp-F 5'-GGGGTCTGGACACCCTATCT-3', Agrp-R 5'CACACGTGACTGCTTCCTGT-3' $)^{78}$. Fold enrichment was calculated relative to the no-antibody control samples.

RNAscope. WT female C57BL6 mice were anesthetized with Pentobarbital then transcardially perfused with 20 $\mathrm{ml}$ PBS followed by $40 \mathrm{ml}$ 10\% Formalin/PBS (Sigma). Brains were removed and incubated in $15 \%$ sucrose/10\% Formalin overnight at $4^{\circ} \mathrm{C}$. Following cryoprotection in $30 \%$ sucrose/PBS, brains were embedded in OCT on dryice and stored at $-80^{\circ} \mathrm{C}$. Serial $20 \mu \mathrm{m}$ sections were cut using a cryostat and mounted on glass slides (VWR) and sections stained with RNAscope $®$ Probe - Mm-Nr5a2-O1-C2 (cat number 547841-C2), Agrp RNAscope ${ }^{\circ}$ Probe - Mm-Agrp (cat number 400711).

\section{RESULTS}

Olanzapine treatment results in significant elevation of NR5a2 and Agrp in hypothalamic cell lines. OLZ treatment of hypothalamic cells resulted in a significant dose-dependent upregulation of both Nr5a2 (Fig. 1A) and Agrp genes expression (Fig. 1B) compared to vehicle treatment. While in general AP-drugs drive hyperphagia and weight gain, there is significant variation in the magnitude of these effects ${ }^{79}$. In our recent study, ${ }^{29}$ we measured antipsychotic-induced weight gain (AIWG) in mice and stratified into subgroups that were highly prone to weight gain (gained $6.3 \mathrm{~g}$ body weight) and weight gain resistant (gained $1.3 \mathrm{~g}$ body weight) ${ }^{29}$. In addition to the previously noted elevation in the hypothalamic expression of Agrp, we also observed a highly significant elevation of Agrp (Fig. 1C) and NR5a2 (Fig. 1D) expression in the AIWG-prone compared with the AIWG-resistant mice. In addition, AIWG-prone mice were also hyperphagic compared to AIWG-resistant mice and thus the elevated hypothalamic expression of NR5a2 and Agrp in the AIWG-prone mice further suggests that these genes may play a role in AP-induced hyperphagia and weight gain.

\section{NR5a2 inhibitor treatment reduces AP-induced food intake and weight gain.}

We further investigated the role of $\mathrm{Nr} 5 \mathrm{a} 2$ in AP-induced hyperphagia in mice using a specific $\mathrm{Nr} 5 \mathrm{a} 2$ antagonist $\left(\mathrm{SR} 1848\right.$, IP $30 \mathrm{mg} / \mathrm{kg}$ daily) ${ }^{70}$. SR1848 inhibits NR5a2 function by triggering translocation of Nr5a2 from the nucleus to the cytoplasm, which ultimately abrogates its ability to transduce transcription of its targets ${ }^{70}$. As expected, OLZ treatment resulted in elevated hypothalamic expression of NR5a2 and co-treatment with SR1848 (OLZ+SR) did not impact NR5a2 expression levels (Fig. 2A). However, co-treatment of OLZ with SR1848 resulted in significantly reduced daily food intake (Fig. 2B) and weight gain (Fig. 2C) compared with OLZ alone over 7 days of treatment. Furthermore, hypothalamic levels of Agrp (Fig. 2D) were significantly reduced by cotreatment, while other appetite regulating neuropeptides Npy and Pomc levels were not significantly changed. To determine whether SR1848 has a direct effect on hypothalamic gene expression, we treated hypothalamic cell lines with SR1848 and measured Agrp gene expression (Fig. 2E). We observed significant reduction in Agrp expression levels after either 1uM or 5uM SR1848 dosing for 6 hours suggesting inhibition of NR5a2 in the hypothalamus has a direct impact on Agrp gene expression.

\section{The knockdown of $\mathrm{Nr} 5 \mathrm{a} 2$ in the arcuate nucleus partially reversed olanzapine-induced hyperphagia and} weight gain.

To determine whether AP-induced food intake and body weight regulation in vivo require the expression of $\mathrm{Nr} 5 \mathrm{a} 2$ specifically in the hypothalamus, we used siRNA-mediated knockdown of Nr5a2 expression in the hypothalamus (Fig. 3A). As expected, Nr5a2 expression was increased by OLZ treatment, and treatment with siRNA targeting NR5a2 significantly reduced NR5a2 expression (Fig. 3A). OLZ treatment increased food intake (Fig. 3B) and body weight gain (Fig. 3C-D) which was reversed by hypothalamic NR5a2 siRNA treatment. Furthermore, gonadal (gWAT) and subcutaneous (sWAT) fat mass were also significantly elevated by OLZ treatment and significantly reduced by NR5a2 siRNA compared to siRNA control (Fig. 3E).

\section{Genetic deletion of Agrp in mice prevented olanzapine-induced hyperphagia and weight gain.}

Since OLZ treatment increases the expression of Agrp similarly to Nr5a2, we tested whether Agrp is necessary for the hyperphagic effect of OLZ. As expected, OLZ treatment of WT mice induced higher food intake (Fig. 4A) and weight gain (Fig.4B-C) compared with control-treated mice. On the other end, AgrpKO mice were resistant to the hyperphagic and weight gain response to OLZ treatment (Fig.4A-C). While OLZ treatment resulted in 
elevated hypothalamic transcriptional levels of $N R 5 a 2$ in AgrpKO mice compared with control treated KO mice, the expression of Npy, Pomc and Cart was unchanged, (Fig 4.D) suggesting NR5a2 maybe upstream of Agrp regulation.

\section{NR5a2 directly regulated Agrp expression}

These data led us to hypothesize that NR5a2 may directly regulate the expression of Agrp by binding to its promoter. To test this, we conducted chromatin immunoprecipitation with NR5a2 antibodies followed by PCR (ChIP-PCR) in hypothalamic mHypo-59A cells. In agreement with previous studies in neuronal stem cells, ${ }^{61}$ we found that Nr5a2 binds the Prospero Homeobox 1(Prox1) promoter (Fig. 5A). We then used primers specific for Agrp promoter region ${ }^{78}$ and determined $\sim 2$-fold enrichment of Nr5a2 binding to the Agrp promoter region over the control sample (Fig.5B). These ChIP experiments in hypothalamic cells identify Agrp as a direct transcriptional target of the transcription factor NR5a2. In situ hybridization analysis determined that NR5a2 is expressed highly and specifically in the ARC of the hypothalamus (Fig. 5C). Co-staining with Agrp revealed that a subset of NR5a2 expressing neurons also contain Agrp (Fig. 5C). Therefore, these studies suggest that a refined population of NR5a2-expressing cells co-express Agrp in the ARC which play a major role in AP-induced hyperphagia and weight gain.

\section{DISCUSSION}

In these studies, we used several mouse models to investigate the role of Nr5a2 in AP-induced food intake and weight gain. We first determined that OLZ treatment resulted in a dose-dependent increase in both Nr5a2 and Agrp expression in hypothalamic cells. Furthermore, hypothalamic Nr5a2 expression was highly induced in mice that were particularly prone to AIWG compared with mice that were relatively protected from AIWG. Administration of SR1848, a specific Nr5a2 inhibitor, decreased OLZ-induced hyperphagia and weight gain and knockdown of $\mathrm{Nr} 5 \mathrm{a} 2$ in the ARC partially reversed OLZ-induced hyperphagia. Importantly, Agrp null mice were protected from OLZ-induced hyperphagia and weight gain, despite having elevated hypothalamic Nr5a2 expression, suggesting this transcription factor may regulate Agrp expression. The ChIP-PCR results reported in the current study show, for the first time, that NR5a2 directly binds to the Agrp promoter region and suggest that $\mathrm{Nr} 5 \mathrm{a} 2$ directly regulates the expression of this pro-feeding neuropeptide in the hypothalamus. Our in-situ studies also confirmed that $\mathrm{Nr} 5 \mathrm{a} 2$ and Agrp are co-localized in a small subset of cells in the ARC and this coexpression is also further supported by single cell RNA seq studies of the ARC ${ }^{65}$.

Despite the importance of Agrp in the homeostatic control of feeding, the transcriptional regulation of its expression is still poorly understood. Studies have shown Agrp transcription is regulated by key energy sensors, including Peroxisome proliferator-activated receptor gamma coactivator 1-alpha ${ }^{80}$, AMP-activated protein kinase or sirtuin 1, and Estrogen receptor alpha and Signal transducer and activator of transcription $3^{81}$, forkhead box protein $01^{82}$, Krüppel-like factor $4^{83}$. Our studies discovered a new transcriptional regulator $\mathrm{Nr} 5 \mathrm{a} 2$ to this important list of factors that can regulate Agrp expression. Future chIP-seq studies are warranted to determine the comprehensive transcriptional targets of $\mathrm{Nr} 5 \mathrm{a} 2$ in Agrp-expressing neurons. Given that $\mathrm{Nr} 5 \mathrm{a} 2$ is also expressed in non-Agrp expressing cells, it will be important to investigate its transcriptional targets in other neuronal population in the ARC.

$\mathrm{Nr} 5 \mathrm{a} 2$ has recently been implicated as playing an important role in maintenance of neuronal differentiation and identity in the hippocampus. In these studies, deletion of Nr5A2 in the dentate gryrus cells in vivo lead to a reduction of the number of NeuN as well as Calbindin-positive neurons ${ }^{84}$. Similar studies in the hypothalamus will be necessary to reveal if this a broader function of NR5a2 in mammalian brain function and plasticity.

To enable transcription factors to bind, chromatin must be an 'open state' and these accessible regions can be determined using a technique called Assay for Transposase-Accessible Chromatin combined with sequencing (ATAC-seq). ATAC seq studies from the human prefrontal cortex found enriched motifs for NR5a2 target genes in schizophrenia patients (treated with APs) compared with matched case controls ${ }^{85}$. These studies confirm that APs impact NR5a2 function in the human brain and suggest NR5a2 is an important target for future therapeutic development. 
In summary, these studies identify a novel mechanism by which OLZ triggers the transcription of Agrp through NR5a2 in a subset of Agrp neurons to promote hyperphagia. These findings can be used to inform future clinical development of APs that do not activate hyperphagia and provide deep insights into the regulation of eating behavior. Importantly, it is critical to mitigate AP-induced weight gain to prevent patient non-compliance ${ }^{86}$ and avoid further exacerbating the growing obesity epidemic and the associated increase in the prevalence of metabolic diseases.

\section{ACKNOWLEDGMENTS}

This study was accomplished through the support by grants including the National Institutes of Health grant R01DK117872 (OO), the National Institute of Health grant P30 DK063491 (Diabetes Research Center, UCSD) (FT and OO) and Larry L. Hillblom Foundation Postdoctoral Fellowship 2019-D-007-FEL (RZ).

Authors' contribution: RCZ, ZZ, AP, and OO performed in vivo experiments. RCZ, DZ, PMP, AL performed laboratory analyses. SMC and FT provided intellectual input and oversight of technical expertise. RCZ, OO and FT analyzed the data and wrote the manuscript.

\section{Conflict of Interest: None to declare}

\section{References}

1 Moore, T. J. \& Mattison, D. R. Adult Utilization of Psychiatric Drugs and Differences by Sex, Age, and Race. JAMA internal medicine 177, 274-275, doi:10.1001/jamainternmed.2016.7507 (2017).

2 Painter, J. T. et al. Analysis of the Appropriateness of Off-Label Antipsychotic Use for Mental Health Indications in a Veteran Population. Pharmacotherapy 37, 438-446, doi:10.1002/phar.1910 (2017).

3 Wofford, M. R., King, D. S. \& Harrell, T. K. Drug-induced metabolic syndrome. Journal of clinical hypertension 8, 114-119 (2006).

4 Comer, J. S., Mojtabai, R. \& Olfson, M. National trends in the antipsychotic treatment of psychiatric outpatients with anxiety disorders. The American journal of psychiatry 168, 10571065, doi:10.1176/appi.ajp.2011.11010087 (2011).

5 Nesvag, R. et al. The incidence, psychiatric co-morbidity and pharmacological treatment of severe mental disorders in children and adolescents. European psychiatry : the journal of the Association of European Psychiatrists 49, 16-22, doi:10.1016/j.eurpsy.2017.12.009 (2018). Verdoux, H., Tournier, M. \& Begaud, B. Antipsychotic prescribing trends: a review of pharmacoepidemiological studies. Acta psychiatrica Scandinavica 121, 4-10, doi:10.1111/j.16000447.2009.01425.x (2010).

7 Jensen, G. L. Drug-induced hyperphagia: what can we learn from psychiatric medications? JPEN. Journal of parenteral and enteral nutrition 32, 578-581, doi:10.1177/0148607108321708 (2008).

8 Verhaegen, A. A. \& Van Gaal, L. F. Drug-induced obesity and its metabolic consequences: a review with a focus on mechanisms and possible therapeutic options. Journal of endocrinological investigation 40, 1165-1174, doi:10.1007/s40618-017-0719-6 (2017).

9 Bak, M., Fransen, A., Janssen, J., van Os, J. \& Drukker, M. Almost all antipsychotics result in weight gain: a meta-analysis. PloS one 9, e94112, doi:10.1371/journal.pone.0094112 (2014). 
10 Asenjo Lobos, C. et al. Clozapine versus other atypical antipsychotics for schizophrenia. The Cochrane database of systematic reviews, CD006633, doi:10.1002/14651858.CD006633.pub2 (2010).

11 Komossa, K. et al. Olanzapine versus other atypical antipsychotics for schizophrenia. The Cochrane database of systematic reviews, CD006654, doi:10.1002/14651858.CD006654.pub2 (2010).

12 Komossa, K. et al. Quetiapine versus other atypical antipsychotics for schizophrenia. The Cochrane database of systematic reviews, CD006625, doi:10.1002/14651858.CD006625.pub2 (2010).

13 Komossa, K. et al. Risperidone versus other atypical antipsychotics for schizophrenia. The Cochrane database of systematic reviews, CD006626, doi:10.1002/14651858.CD006626.pub2 (2011).

14 Lieberman, J. A. et al. Effectiveness of antipsychotic drugs in patients with chronic schizophrenia. The New England journal of medicine 353, 1209-1223, doi:10.1056/NEJMoa051688 (2005).

15 Musil, R., Obermeier, M., Russ, P. \& Hamerle, M. Weight gain and antipsychotics: a drug safety review. Expert opinion on drug safety 14, 73-96, doi:10.1517/14740338.2015.974549 (2015).

16 Newcomer, J. W. Second-generation (atypical) antipsychotics and metabolic effects: a comprehensive literature review. CNS drugs 19 Suppl 1, 1-93 (2005).

17 Manschreck, T. C. \& Boshes, R. A. The CATIE schizophrenia trial: results, impact, controversy. Harvard review of psychiatry 15, 245-258, doi:10.1080/10673220701679838 (2007).

18 Fountaine, R. J. et al. Increased food intake and energy expenditure following administration of olanzapine to healthy men. Obesity 18, 1646-1651, doi:10.1038/oby.2010.6 (2010).

19 Gothelf, D. et al. Weight gain associated with increased food intake and low habitual activity levels in male adolescent schizophrenic inpatients treated with olanzapine. The American journal of psychiatry 159, 1055-1057, doi:10.1176/appi.ajp.159.6.1055 (2002).

20 Kluge, M. et al. Clozapine and olanzapine are associated with food craving and binge eating: results from a randomized double-blind study. Journal of clinical psychopharmacology 27, 662666, doi:10.1097/jcp.0b013e31815a8872 (2007).

21 Cooper, G. D., Goudie, A. J. \& Halford, J. C. Acute effects of olanzapine on behavioural expression including the behavioural satiety sequence in female rats. Journal of psychopharmacology 24, 1069-1078, doi:10.1177/0269881109102543 (2010).

22 Davoodi, N., Kalinichev, M., Korneev, S. A. \& Clifton, P. G. Hyperphagia and increased meal size are responsible for weight gain in rats treated sub-chronically with olanzapine. Psychopharmacology 203, 693-702, doi:10.1007/s00213-008-1415-1 (2009).

23 Minet-Ringuet, J., Even, P. C., Guesdon, B., Tome, D. \& de Beaurepaire, R. Effects of chronic neuroleptic treatments on nutrient selection, body weight, and body composition in the male rat under dietary self-selection. Behavioural brain research 163, 204-211, doi:10.1016/j.bbr.2005.05.004 (2005).

24 Thornton-Jones, Z., Neill, J. C. \& Reynolds, G. P. The atypical antipsychotic olanzapine enhances ingestive behaviour in the rat: a preliminary study. Journal of psychopharmacology 16, 35-37, doi:10.1177/026988110201600111 (2002).

25 Weston-Green, K., Huang, X. F. \& Deng, C. Olanzapine treatment and metabolic dysfunction: a dose response study in female Sprague Dawley rats. Behavioural brain research 217, 337-346, doi:10.1016/j.bbr.2010.10.039 (2011).

26 Zhang, Q. et al. Hypothalamic ghrelin signalling mediates olanzapine-induced hyperphagia and weight gain in female rats. The international journal of neuropsychopharmacology 17, 807-818, doi:10.1017/S1461145713001697 (2014).

27 Perez-Gomez, A. et al. A phenotypic Caenorhabditis elegans screen identifies a selective suppressor of antipsychotic-induced hyperphagia. Nature communications 9, 5272, doi:10.1038/s41467-018-07684-y (2018). 
28 Wei, H. et al. Dopamine D2 receptor signaling modulates pancreatic beta cell circadian rhythms. Psychoneuroendocrinology 113, 104551, doi:10.1016/j.psyneuen.2019.104551 (2020).

29 Zapata, R. C. \& Osborn, O. Susceptibility of male wild type mouse strains to antipsychoticinduced weight gain. Physiology \& behavior 220, 112859, doi:10.1016/j.physbeh.2020.112859 (2020).

30 Zapata, R. C. et al. Metabolomic profiles associated with a mouse model of antipsychoticinduced food intake and weight gain. Scientific reports 10, 18581, doi:10.1038/s41598-02075624-2 (2020).

31 Lord, C. C. et al. The atypical antipsychotic olanzapine causes weight gain by targeting serotonin receptor 2C. The Journal of clinical investigation 127, 3402-3406, doi:10.1172/JCI93362 (2017). Hilger, E. et al. The effect of orlistat on plasma levels of psychotropic drugs in patients with longterm psychopharmacotherapy. Journal of clinical psychopharmacology 22, 68-70 (2002).

Fink-Jensen, A., Correll, C. U. \& Vilsboll, T. Liraglutide for the Treatment of Antipsychotic DrugInduced Weight Gain-Reply. JAMA psychiatry $\mathbf{7 4}, \quad$ 1173-1174, doi:10.1001/jamapsychiatry.2017.2702 (2017).

Sacchetti, E., Guarneri, L. \& Bravi, D. H(2) antagonist nizatidine may control olanzapineassociated weight gain in schizophrenic patients. Biological psychiatry 48, 167-168 (2000).

Baptista, T. et al. Metformin for prevention of weight gain and insulin resistance with olanzapine: a double-blind placebo-controlled trial. Canadian journal of psychiatry. Revue canadienne de psychiatrie 51, 192-196, doi:10.1177/070674370605100310 (2006). Gomez-Amaro, R. L. et al. Measuring Food Intake and Nutrient Absorption in Caenorhabditis elegans. Genetics 200, 443-454, doi:10.1534/genetics.115.175851 (2015).

37 Huang, X. F., Han, M., Huang, X., Zavitsanou, K. \& Deng, C. Olanzapine differentially affects 5HT2Aand2C receptor mRNA expression in the rat brain. Behavioural brain research 171, 355362, doi:10.1016/j.bbr.2006.03.040 (2006).

38 Morgan, A. P. et al. The antipsychotic olanzapine interacts with the gut microbiome to cause weight gain in mouse. PloS one 9, e115225, doi:10.1371/journal.pone.0115225 (2014). Stefanidis, A. et al. The role of thermogenesis in antipsychotic drug-induced weight gain. Obesity 17, 16-24, doi:10.1038/oby.2008.468 (2009).

40 Zhang, Q. et al. Olanzapine reduced brown adipose tissue thermogenesis and locomotor activity in female rats. Progress in neuro-psychopharmacology \& biological psychiatry 51, 172-180, doi:10.1016/j.pnpbp.2014.02.003 (2014).

41 Ahima, R. S. \& Antwi, D. A. Brain regulation of appetite and satiety. Endocrinology and metabolism clinics of North America 37, 811-823, doi:10.1016/j.ecl.2008.08.005 (2008).

Broberger, C., Johansen, J., Johansson, C., Schalling, M. \& Hokfelt, T. The neuropeptide Y/agouti gene-related protein (AGRP) brain circuitry in normal, anorectic, and monosodium glutamate-treated mice. Proceedings of the National Academy of Sciences of the United States of America 95, 15043-15048 (1998).

43 Hahn, T. M., Breininger, J. F., Baskin, D. G. \& Schwartz, M. W. Coexpression of Agrp and NPY in fasting-activated hypothalamic neurons. Nature neuroscience 1, 271-272, doi:10.1038/1082 (1998).

44 Aponte, Y., Atasoy, D. \& Sternson, S. M. AGRP neurons are sufficient to orchestrate feeding behavior rapidly and without training. Nature neuroscience 14, 351-355, doi:10.1038/nn.2739 (2011).

45 Krashes, M. J. et al. Rapid, reversible activation of AgRP neurons drives feeding behavior in mice. The Journal of clinical investigation 121, 1424-1428, doi:10.1172/JCl46229 (2011).

46 Stanley, B. G., Kyrkouli, S. E., Lampert, S. \& Leibowitz, S. F. Neuropeptide Y chronically injected into the hypothalamus: a powerful neurochemical inducer of hyperphagia and obesity. Peptides 7, 1189-1192 (1986). 
47 Ferno, J. et al. Olanzapine-induced hyperphagia and weight gain associate with orexigenic hypothalamic neuropeptide signaling without concomitant AMPK phosphorylation. PloS one $\mathbf{6}$, e20571, doi:10.1371/journal.pone.0020571 (2011).

48 Martins, P. J., Haas, M. \& Obici, S. Central nervous system delivery of the antipsychotic olanzapine induces hepatic insulin resistance. Diabetes 59, 2418-2425, doi:10.2337/db10-0449 (2010).

49 Miller, C. L. On the mechanism of action of antipsychotic drugs: a chemical reaction not receptor blockade. Current drug discovery technologies 10, 195-208 (2013).

50 Panariello, F., Polsinelli, G., Borlido, C., Monda, M. \& De Luca, V. The role of leptin in antipsychotic-induced weight gain: genetic and non-genetic factors. Journal of obesity 2012, 572848, doi:10.1155/2012/572848 (2012).

51 Shams, T. A. \& Muller, D. J. Antipsychotic induced weight gain: genetics, epigenetics, and biomarkers reviewed. Current psychiatry reports 16, 473, doi:10.1007/s11920-014-0473-9 (2014).

52 Miranda, D. A. et al. LRH-1 regulates hepatic lipid homeostasis and maintains arachidonoyl phospholipid pools critical for phospholipid diversity. JCl insight 3, doi:10.1172/jci.insight.96151 (2018).

53 Stein, S. \& Schoonjans, K. Molecular basis for the regulation of the nuclear receptor LRH-1. Current opinion in cell biology 33, 26-34, doi:10.1016/j.ceb.2014.10.007 (2015).

54 Fayard, E., Auwerx, J. \& Schoonjans, K. LRH-1: an orphan nuclear receptor involved in development, metabolism and steroidogenesis. Trends in cell biology 14, 250-260, doi:10.1016/j.tcb.2004.03.008 (2004).

55 Mrosek, N. et al. Transcriptional regulation of adipocyte formation by the liver receptor homologue 1 (Lrh1)-Small hetero-dimerization partner (Shp) network. Molecular metabolism 2, 314-323, doi:10.1016/j.molmet.2013.03.003 (2013).

56 Fernandez-Marcos, P. J., Auwerx, J. \& Schoonjans, K. Emerging actions of the nuclear receptor $\mathrm{LRH}-1$ in the gut. Biochimica et biophysica acta 1812, 947-955, doi:10.1016/j.bbadis.2010.12.010 (2011).

57 Cobo, l. et al. Transcriptional regulation by NR5A2 links differentiation and inflammation in the pancreas. Nature 554, 533-537, doi:10.1038/nature25751 (2018).

58 Lee, Y. K. et al. Liver receptor homolog-1 regulates bile acid homeostasis but is not essential for feedback regulation of bile acid synthesis. Molecular endocrinology 22, 1345-1356, doi:10.1210/me.2007-0565 (2008).

59 Sablin, E. P., Krylova, I. N., Fletterick, R. J. \& Ingraham, H. A. Structural basis for ligandindependent activation of the orphan nuclear receptor LRH-1. Molecular cell 11, 1575-1585 (2003).

60 Grgurevic, N., Tobet, S. \& Majdic, G. Widespread expression of liver receptor homolog 1 in mouse brain. Neuro endocrinology letters 26, 541-547 (2005).

61 Stergiopoulos, A. \& Politis, P. K. Nuclear receptor NR5A2 controls neural stem cell fate decisions during development. Nature communications 7, 12230, doi:10.1038/ncomms12230 (2016).

62 Atkin, S. D. et al. Nuclear receptor LRH-1 induces the reproductive neuropeptide kisspeptin in the hypothalamus. Molecular endocrinology 27, 598-605, doi:10.1210/me.2012-1371 (2013).

63 Gofflot, F. et al. Systematic gene expression mapping clusters nuclear receptors according to their function in the brain. Cell 131, 405-418, doi:10.1016/j.cell.2007.09.012 (2007).

64 Higashiyama, H., Kinoshita, M. \& Asano, S. Expression profiling of liver receptor homologue 1 $(\mathrm{LRH}-1)$ in mouse tissues using tissue microarray. Journal of molecular histology 38, 45-52, doi:10.1007/s10735-007-9077-6 (2007).

65 Campbell, J. N. et al. A molecular census of arcuate hypothalamus and median eminence cell types. Nature neuroscience 20, 484-496, doi:10.1038/nn.4495 (2017).

66 Oosterveer, M. H. et al. LRH-1-dependent glucose sensing determines intermediary metabolism in liver. The Journal of clinical investigation 122, 2817-2826, doi:10.1172/JCI62368 (2012). 
67 Cobo-Vuilleumier, N. et al. LRH-1 agonism favours an immune-islet dialogue which protects against diabetes mellitus. Nature communications 9, 1488, doi:10.1038/s41467-018-03943-0 (2018).

68 Chen, X. et al. Olanzapine increases AMPK-NPY orexigenic signaling by disrupting H1RGHSR1a interaction in the hypothalamic neurons of mice. Psychoneuroendocrinology 114, 104594, doi:10.1016/j.psyneuen.2020.104594 (2020).

69 Dalvi, P. S., Nazarians-Armavil, A., Tung, S. \& Belsham, D. D. Immortalized neurons for the study of hypothalamic function. American journal of physiology. Regulatory, integrative and comparative physiology 300, R1030-1052, doi:10.1152/ajpregu.00649.2010 (2011).

70 Corzo, C. A. et al. Antiproliferation activity of a small molecule repressor of liver receptor homolog 1. Molecular pharmacology 87, 296-304, doi:10.1124/mol.114.095554 (2015).

$71 \mathrm{Li}, \mathrm{B}$. et al. Identification of optimal reference genes for RT-qPCR in the rat hypothalamus and intestine for the study of obesity. International journal of obesity 38, 192-197, doi:10.1038/ijo.2013.86 (2014).

72 Cameron, K. M. \& Speakman, J. R. The extent and function of 'food grinding' in the laboratory mouse (Mus musculus). Laboratory animals 44, 298-304, doi:10.1258/la.2010.010002 (2010).

73 Fields, J., Swinton, M., Montilla-Perez, P., Ricciardelli, E. \& Telese, F. The Cannabinoid Receptor Agonist, WIN, Suppresses the Activation of Proinflammatory Genes Induced by Interleukin 1 Beta in Human Astrocytes. Cannabis and Cannabinoid Research, doi:https://www.liebertpub.com/doi/10.1089/can.2020.0128 (2020).

$74 \mathrm{Ma}, \mathrm{Q}$. \& Telese, F. Genome-wide epigenetic analysis of MEF2A and MEF2C transcription factors in mouse cortical neurons. Commun Integr Biol 8, e1087624, doi:10.1080/19420889.2015.1087624 (2015).

75 Wang, J. et al. LSD1n is an H4K20 demethylase regulating memory formation via transcriptional elongation control. Nature neuroscience 18, 1256-1264, doi:10.1038/nn.4069 (2015).

Chong, H. K., Biesinger, J., Seo, Y. K., Xie, X. \& Osborne, T. F. Genome-wide analysis of hepatic $\mathrm{LRH}-1$ reveals a promoter binding preference and suggests a role in regulating genes of lipid metabolism in concert with FXR. BMC Genomics 13, 51, doi:10.1186/1471-2164-13-51 (2012).

77 Milona, A. et al. Steroidogenic control of liver metabolism through a nuclear receptor-network. Molecular metabolism 30, 221-229, doi:10.1016/j.molmet.2019.09.007 (2019).

78 Lee, B. et al. Brain-specific homeobox factor as a target selector for glucocorticoid receptor in energy balance. Molecular and cellular biology 33, 2650-2658, doi:10.1128/MCB.00094-13 (2013).

79 Zapata, R. C. et al. Conserved immunomodulatory transcriptional networks underlie antipsychotic-induced weight gain. Translational psychiatry 11, 405, doi:10.1038/s41398-02101528-y (2021).

80 Gill, J. F., Delezie, J., Santos, G. \& Handschin, C. PGC-1alpha expression in murine AgRP neurons regulates food intake and energy balance. Molecular metabolism 5, 580-588, doi:10.1016/j.molmet.2016.05.008 (2016).

81 Benedusi, V. et al. Liver ERalpha regulates AgRP neuronal activity in the arcuate nucleus of female mice. Scientific reports 7, 1194, doi:10.1038/s41598-017-01393-0 (2017).

82 Kitamura, T. et al. Forkhead protein FoxO1 mediates Agrp-dependent effects of leptin on food intake. Nature medicine 12, 534-540, doi:10.1038/nm1392 (2006).

83 Ilnytska, O. et al. Molecular mechanisms for activation of the agouti-related protein and stimulation of appetite. Diabetes 60, 97-106, doi:10.2337/db10-0172 (2011).

84 Tsampoula, M. et al. Nuclear Receptor NR5A2 Promotes Neuronal Identity in the Adult Hippocampus. Mol Neurobiol 58, 1952-1962, doi:10.1007/s12035-020-02222-8 (2021).

85 Bryois, J. et al. Evaluation of chromatin accessibility in prefrontal cortex of individuals with schizophrenia. Nature communications 9, 3121, doi:10.1038/s41467-018-05379-y (2018).

86 Tschoner, A. et al. Metabolic side effects of antipsychotic medication. International journal of clinical practice 61, 1356-1370, doi:10.1111/j.1742-1241.2007.01416.x (2007). 


\section{FIGURE LEGENDS}

Figure 1. OLZ treatment is associated with elevated Nr5a2 expression. OLZ treatment of hypothalamic cells results in dose dependent increase in expression of (A) Nr5a2 and (B) Agrp. Mice that are highly Prone to Antipsychotic-Induced Weight Gain (AIWG-P) have significantly elevated hypothalamic levels of (C) $N r 5 a 2$ and (D) Agrp compared with AIWG-Resistant mice (AIWG-R). Data is expressed as mean \pm SEM and was analyzed using one-way ANOVA followed by uncorrected Fisher's LSD test, (A-B, ( $n=3-8$ replicates per group) or students t-test (C-D, $\mathrm{n}=8-10$ replicates per group), * denotes statistical significance at $p<0.05$.

Figure 2. Systemic Nr5a2 antagonist treatment reduces food intake and weigh gain in mice treated with Olanzapine. A) Nr5a2 expression, (B) Average daily food intake, (C) Weight gain, (D) Hypothalamic neuropeptide expression in C57BL/6 WT female mice fed either control diet (CON) or OLZ diet and injected with NR5a2 antagonist (SR1848, 'SR', 30mg/kg) or vehicle (VEH) for 7 days. E) Quantitative PCR determination of Agrp expression in hypothalamic cells lines treated with SR1848 (1uM or 5uM) for 6 hours. Data is expressed as mean \pm SEM and was analyzed using one-way ANOVA followed by uncorrected Fisher's LSD test, * denotes statistical significance at $p<0.05, \mathrm{n}=4-11$ replicates per group.

Figure 3. Hypothalamic knockdown of Nr5a2 significantly bunts OLZ-induced food intake and weight gain. siRNA mediated knock down of $\mathrm{Nr} 5 \mathrm{a} 2$, delivered by stereotaxic injection to the arcuate nucleus, results in (A) reduced expression of Nr5a2, (B) blunted OLZ-induced food intake and (C-D) reduced OLZ-induced weight gain and body fat (E), Data is expressed as mean \pm SEM and was analyzed using one-way ANOVA followed by uncorrected Fisher's LSD test, * denotes statistical significance at $p<0.05, \mathrm{n}=4-5$ per group.

Figure 4. Agrp KO mice are resistant to OLZ-induced hyperphagia and weight gain. A) Food intake (B-C) weight gain, (D) hypothalamic gene expression in WT and KO mice treated with CON or OLZ diets. Data is expressed as mean \pm SEM and was analyzed using one-way ANOVA followed by uncorrected Fisher's LSD test, * denotes statistical significance at $p<0.05, \mathrm{n}=9-12$ per group.

Figure 5. Agrp is a direct transcriptional target of Nr5a2. Chromatin immunoprecipitation with $\mathrm{Nr} 5 \mathrm{a} 2$ antibodies followed by PCR (chIP-PCR) in the hypothalamic cell line (mHypo-59A) result in enrichment of binding to the (A) Prox1 promoter (positive control) and (B) Agrp promoter region compared with beads. Data is expressed as mean \pm SEM and was analyzed using students t-test, ( $n=3$ replicates per group), ${ }^{*}$ denotes statistical significance at $p<0.05$. C. In-situ hybridization reveals NR5a2 (red) is specifically localized to the arcuate nucleus (Agrp, green) and co-expressed in a subset of Agrp neurons (yellow) in the dorsal (D) and Lateral (L) ARC.

Table 1.

\begin{tabular}{|c|c|}
\hline Gene & Primer sequence 5'-3' \\
\hline Agrp & $\begin{array}{c}\text { F: GGAACAGTGTTTTCTGCTCCC } \\
\text { R: ACTCGTGCAGCCTTACACAG }\end{array}$ \\
\hline Npy & $\begin{array}{c}\text { F: TAACAAGCGATGGGGCTGT } \\
\text { R: TTCAAGCCTTGTTCTGGGGG }\end{array}$ \\
\hline Pomc & $\begin{array}{c}\text { F: GGCGACGGAAGAGAAAAGAGG } \\
\text { R: TGTTCAGTCTCCTGCCTGTCG }\end{array}$ \\
\hline Cart & $\begin{array}{c}\text { F: TGGATGATGCGTCCCATG } \\
\text { R: TACTTCTTCTCATAGATCGGAAT }\end{array}$ \\
\hline Nr5a2 & $\begin{array}{c}\text { F: AGTCTGAGGTTCCTTCCCAAAG } \\
\text { R: CTAGAGCAAGCTTCCAGGGG }\end{array}$ \\
\hline Pgk1 & $\begin{array}{c}\text { F: CTGACTTTGGACAAGCTGGACG } \\
\text { R: GCAGCCTTGATCCTTTGGTTG }\end{array}$ \\
Hrpt1 & $\begin{array}{l}\text { F: CACAGGACTAGAACACCTGC } \\
\text { R: GCTGGTGAAAAGGACCTCT }\end{array}$ \\
\hline
\end{tabular}


A

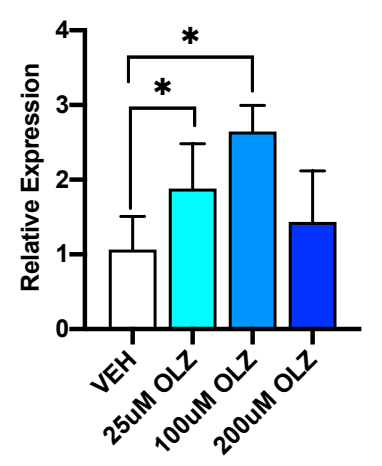

C

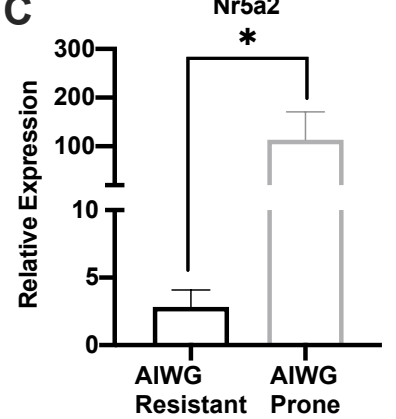

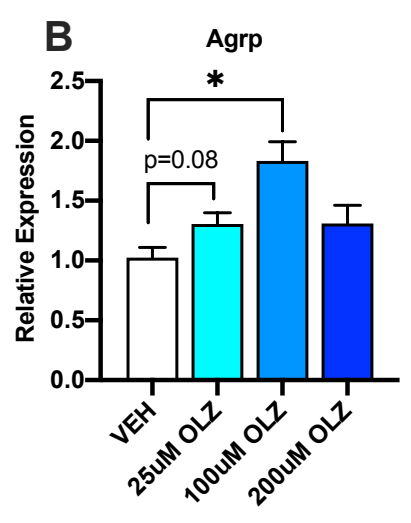

Figure 1

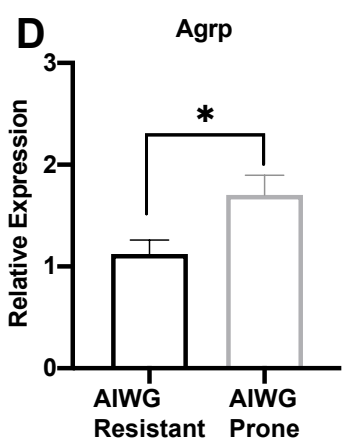


A

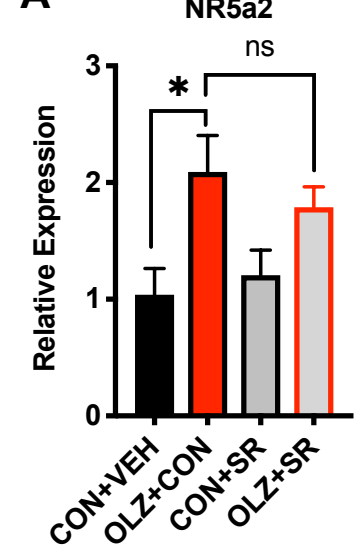

B

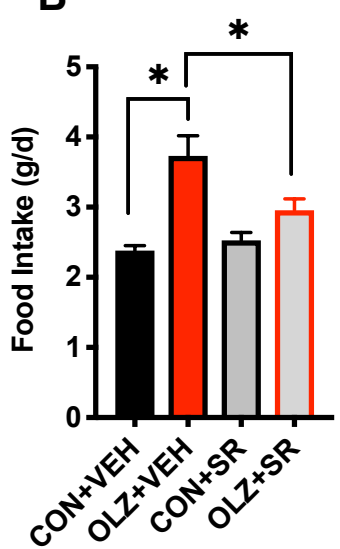

C

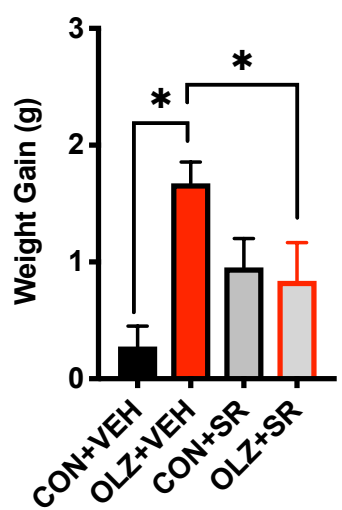

D

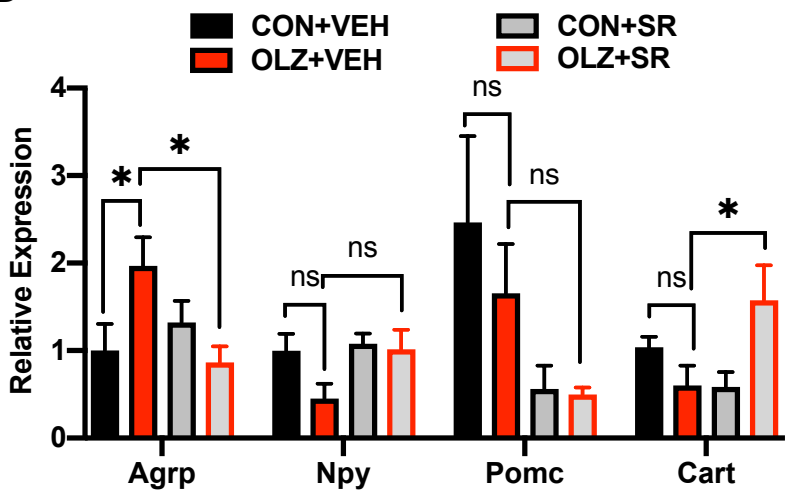

E

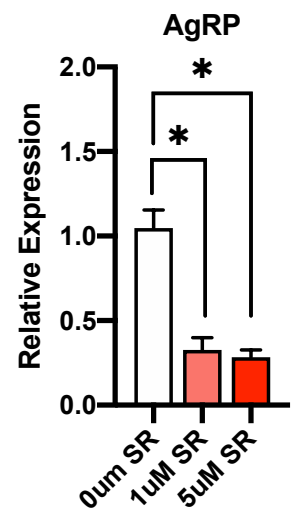


A

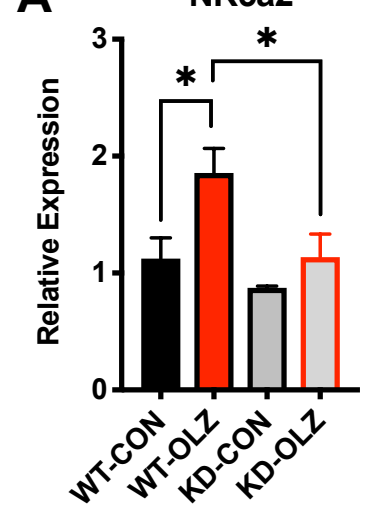

B

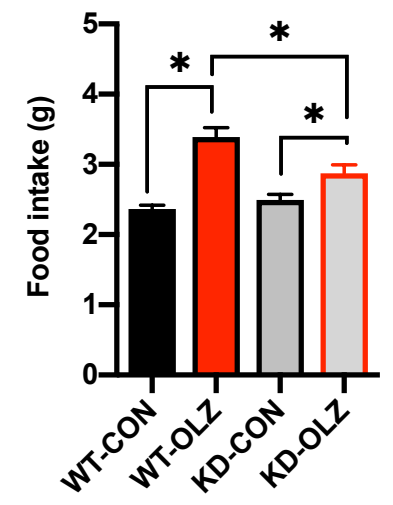

C

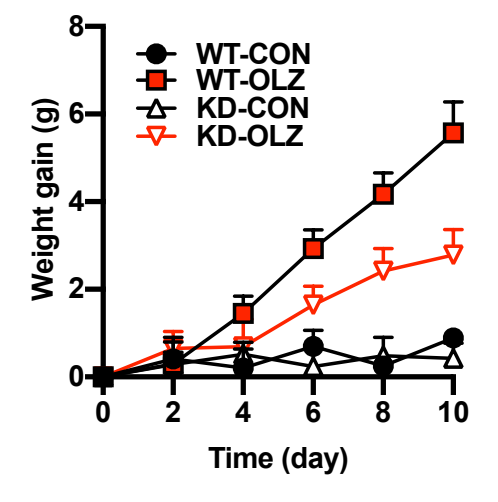

D

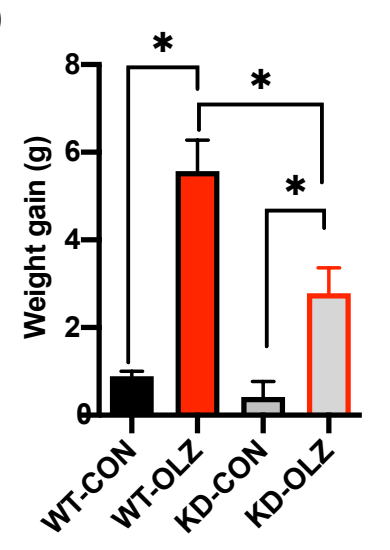

E

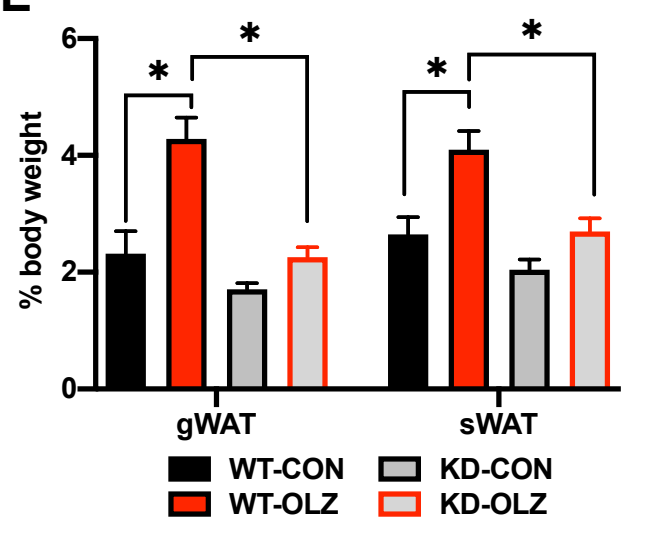


A
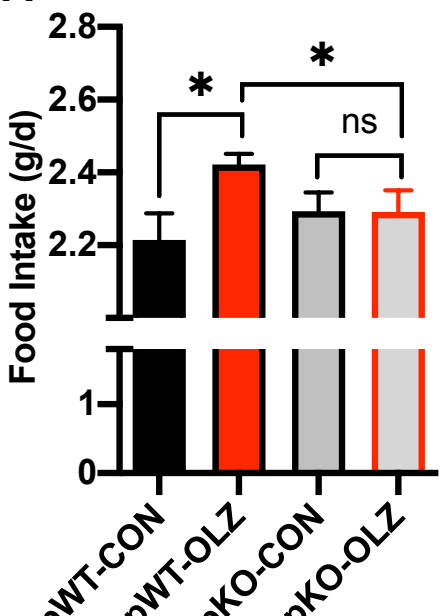

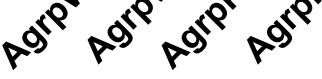

B

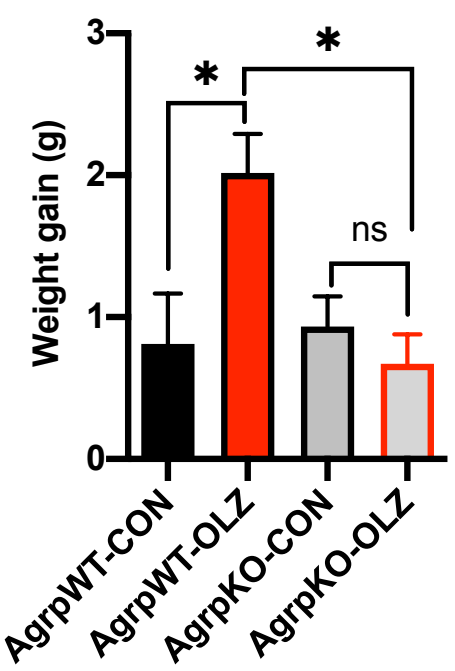

C

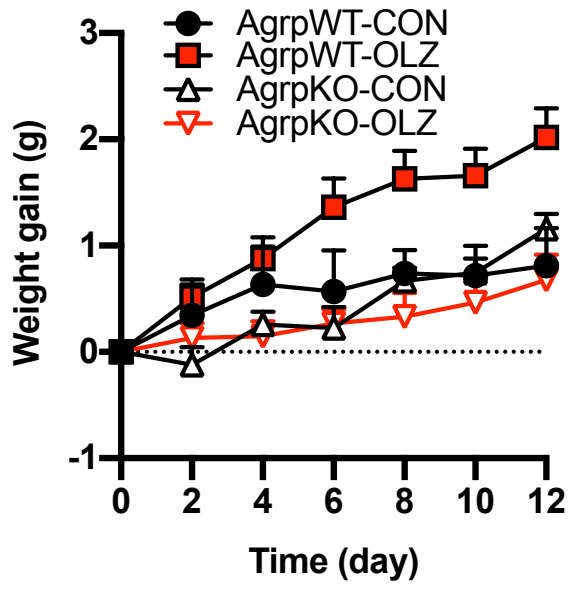

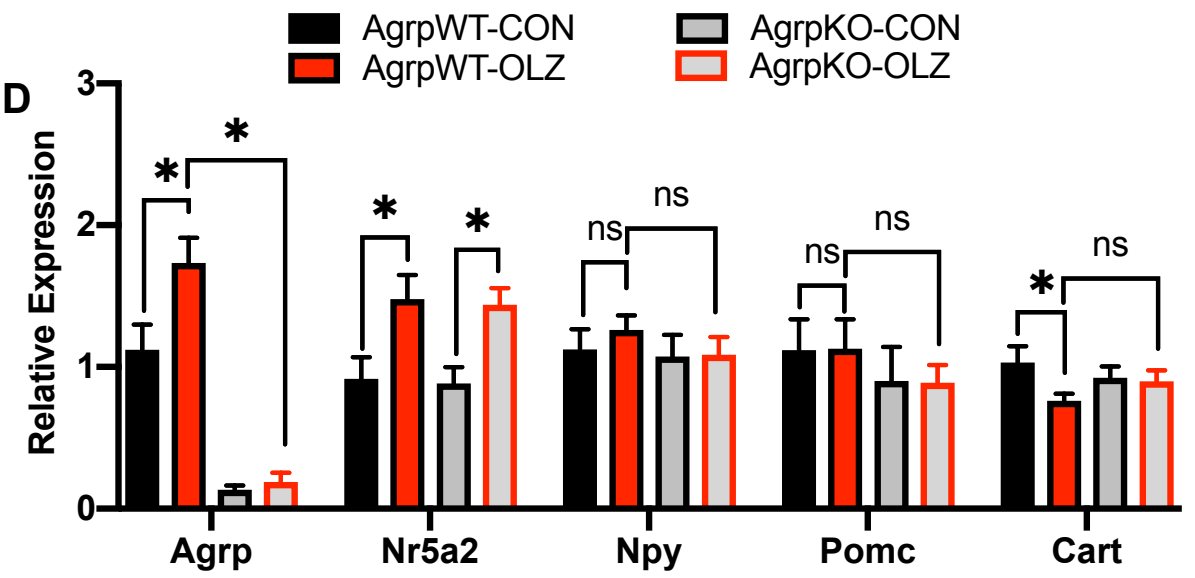


A

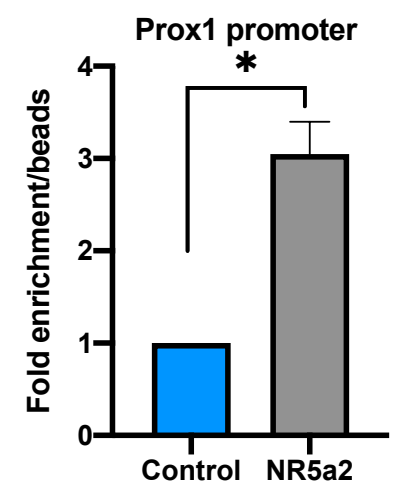

B

Agrp promoter

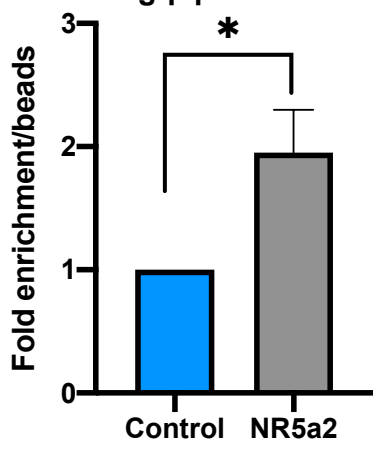

C
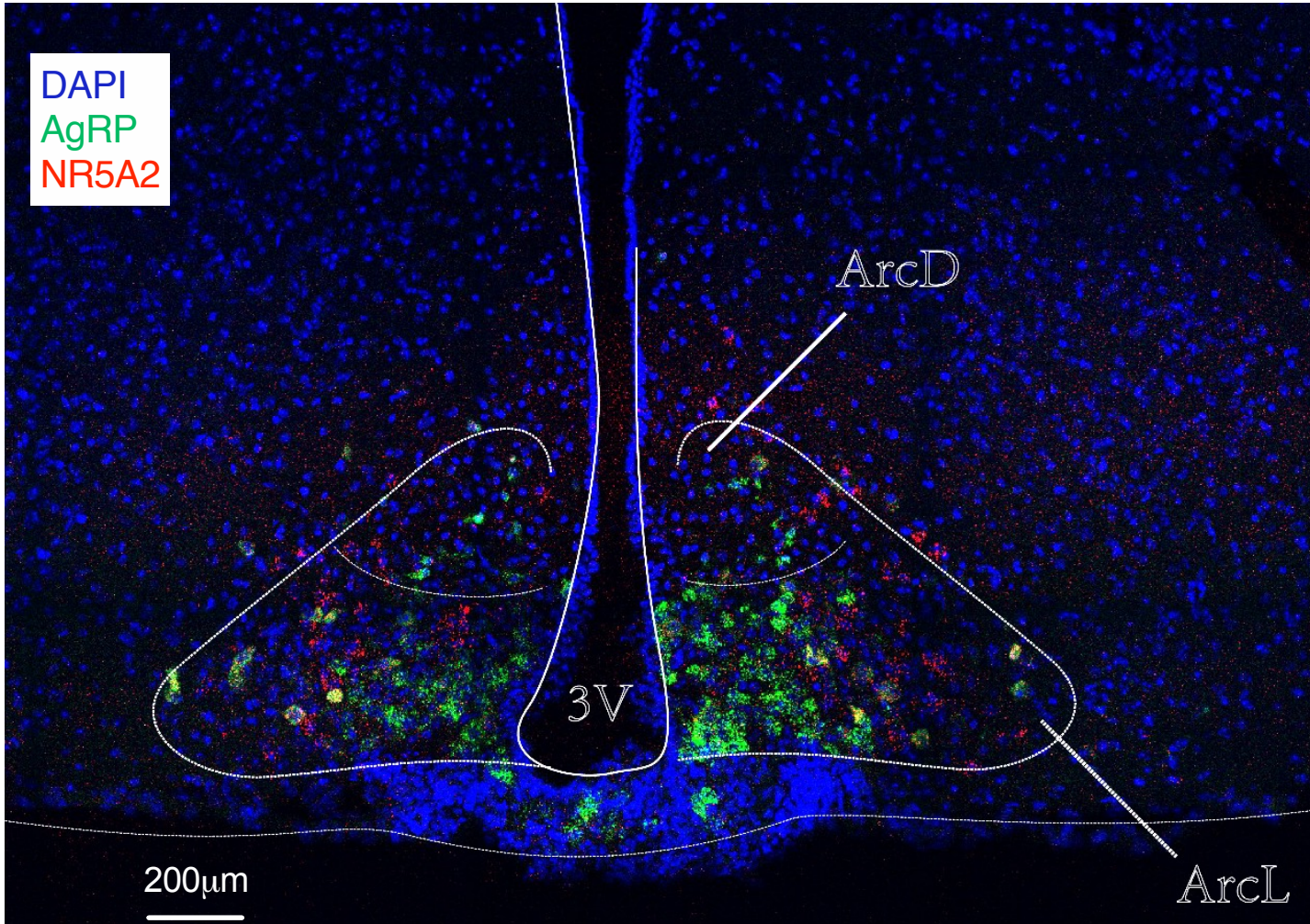